\title{
Alerte aux conférences prédatrices !
}

Quelques traces électroniques laissées dans le monde académique suffisent pour recevoir des sollicitations à publier article ou « numéro spécial » dans un journal inconnu ou pour être « invité " à une conférence lointaine. Le gâchis induit par ces journaux et conférences « prédatrices » n'est pas seulement financier. Fréquemment, des collègues trop peu vigilants se retrouvent à des « conférences » ineptes. D'où cette mise en garde.

\section{Les journaux prédateurs}

Lorsque les journaux scientifiques étaient imprimés, le client était un bibliothécaire, lui même à l'écoute des demandes des scientifiques. La naissance d'une nouvelle revue dépendait du choix d'une communauté. Depuis, une économie nouvelle est apparue, où l'auteur paie pour publier en ligne. Si certains de ces journaux sont incontestables (par exemple Phys. Rev. X), d'autres [1, 2] ne fournissent pas, malgré leurs dires, l'indispensable critique par les pairs. Ils accueillent couramment " résultats " infondés, sans originalité, et purs plagiats. C'est un bibliothécaire, Jeffrey Beall, qui, notant l'éclosion incontrôlée de ce type de revues en ligne, a trouvé l'expression "revue prédatrice ".

Soumettre un travail scientifique original à une revue nouvelle s'avérant prédatrice est rare, mais cauchemardesque. Lorsque l'auteur découvre que la publication sera "invisible ", le retrait est impossible, ou autorisé seulement au prix d'un supplément extravagant : le " copyright ", exigé très en amont, a verrouillé le processus. En fait, la cible commerciale de ces journaux prédateurs est le «tiers-monde » scientifique, où disposer d'une liste de publications à apparence "internationale " est bénéfique.

\section{Les conférences prédatrices}

Les conférences prédatrices [2-5], plus récentes, sont aussi une conséquence de l'Internet et de la gratuité de l'adressage électronique. Elles «vendent " de même des rencontres fourre-tout, sans contrôle scientifique, souvent d'apparence périodique, et sont organisées par de prétendues sociétés « savantes» ou "Académies ». L'objectif est d'attirer des participants payants : les chercheurs sont gratifiés d'un statut d'" orateur invité " (payant) et, à la clientèle spontanée, on promet la gloire d'une sérieuse sélection (" au moins deux ou trois rapporteurs "), là où toute soumission payée sera acceptée. Les frais d'inscription augmentent pour être contributeur plutôt qu'auditeur, et plus encore pour des présentations multiples. On y invente la "présentation virtuelle » : l'envoi d'une vidéo suffit à être considéré comme "participant ". De plus, pour démultiplier le nombre d'" orateurs ", on multiplie les sessions parallèles.

Alors que la nécessité d'actes décline depuis l'échange instantané d'informations, ces conférences persistent à en éditer. Allier tourisme et «publication » (surtout, hélas, dans de vraies " éditions savantes ») est un argument de vente séducteur.
Identifier ces rencontres, à l'affluence variable, est presque un jeu d'enfant : titre grandiose (" $\mathrm{N}^{\text {th }}$ International Conference"), thématiques attrape-tout, comité pléthorique et peu identifiable, incluant beaucoup d'institutions " exotiques ", voire de pure invention [5]. Très longtemps, le programme est " en construction". Des éditions précédentes, ne restent que de jolies photos, garantissant que l'événement a eu lieu. Un même lieu peut réunir des conférences diverses, qui fusionnent in fine, d'où des interventions et un public totalement hétéroclites. Repérer ces jumelages ne peut se faire qu'avec les sites de l'industrie touristique, faisant la publicité (gratuite) des conférences de toute nature. Ces parodies de conférence sont nombreuses en Asie, mais France et Europe ne sont pas épargnées.

\section{Qui assiste et qui organise ?}

Comme pour les journaux prédateurs, la clientèle principale est issue de régions périphériques. Des collègues scientifiquement marginalisés peuvent aussi y "publier » leurs élucubrations autour des grandes théories [6].

Étonnamment, il est fréquent que des collègues de bon renom se laissent abuser par le titre de «conférencier invité " (fig. 1). À leur décharge, l'annonce prétendait à la participation (non confirmée) de célébrités, ou le "Comité " avait été étoffé avec des CVs volés (fig. 1). Certains chercheurs reconnaissent qu'ils auraient pu être plus méfiants (fig. 1) ; d'autres osent plaider : "On sait bien que les invitations, c'est toujours par copinage. "L'intervention de ces collègues, si travaillée soit-elle, sera sans impact face à un public disparate et médiocre.

Les organisateurs réels, bien moins nombreux que le "Comité " fantôme, sont à l'image du public : universités obscures, orateurs dits «invités " ou "pléniers " qui ne sont que «enseignants associés " ou pas encore docteurs. On peut même identifier une poignée d'habitués, auteurs de "keynote talks" répétitifs. Quelques collègues de France ou d'Europe, qui passent pour d'honnêtes spécialistes, se complaisent à "éditer ", hors de leur champ disciplinaire, des "Actes " de ces rencontres. Ces activités peu reluisantes seront inconnues des collègues proches. Pour ces imprudents, le bénéfice matériel n'excède sans doute pas un séjour indu, pas toujours exotique.

Faut-il croire que c'est pour l'entourage (famille, collègues d'autres disciplines...) que ces participations rehaussent le prestige social d'une carrière terne? 
J'ai bien reçu votre dernier courrier.

concernant les accusations portées contre $x x x x$, je n'ai personnellement aucun élément de réponse à vous apporter, et il aurait été grandement préférable de le contacter dírectement afin qu'il puisse y répondre lui-même.

cette façon de procéder jette en effet un soupçon sur certaines activités de ce collègue français, de nature à gravement impacter ses conditions de travail à $x \times x x$

Les documents sont actuellement dans les mains du service juridique de $x \times x \times x$.

\section{cordialement}

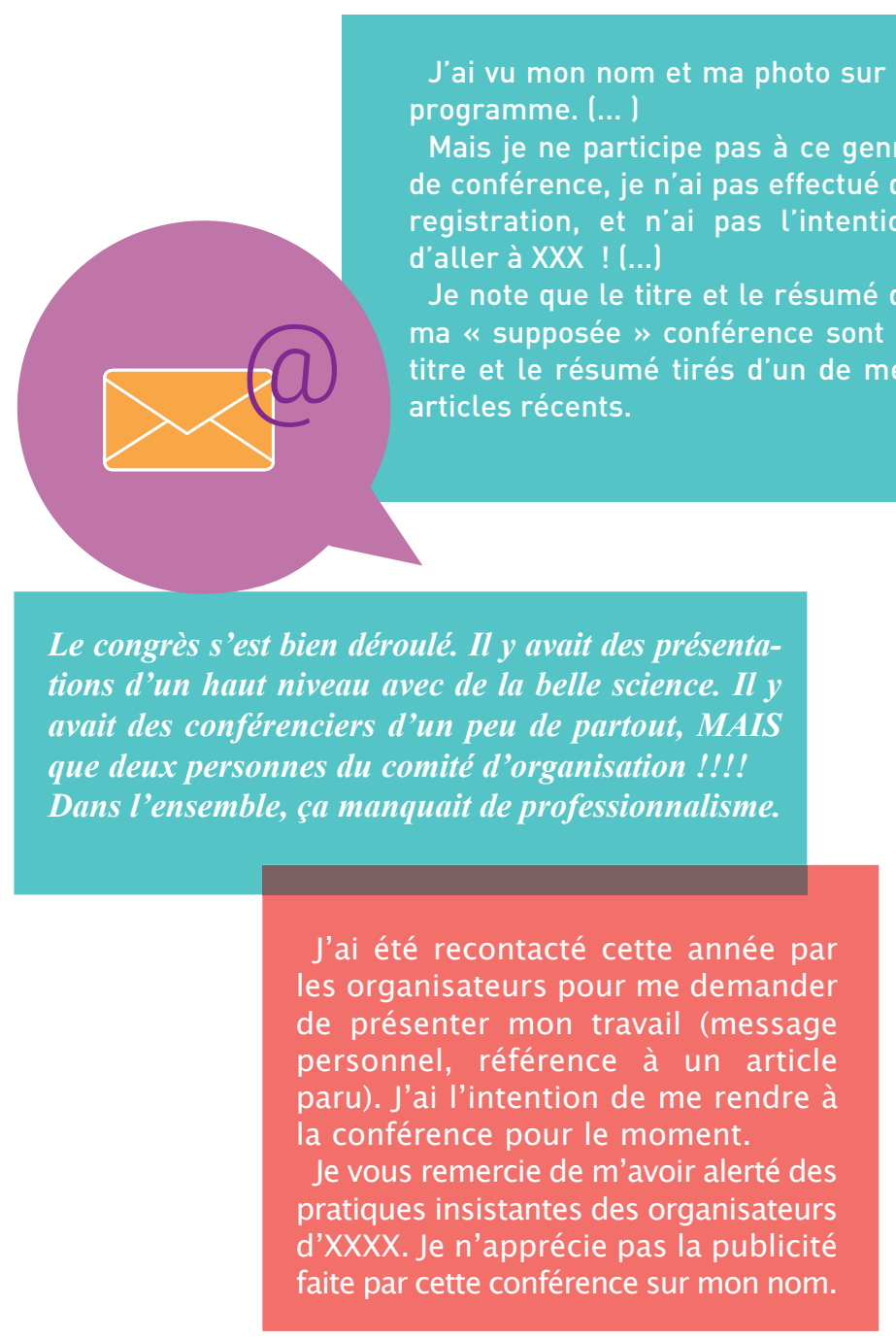

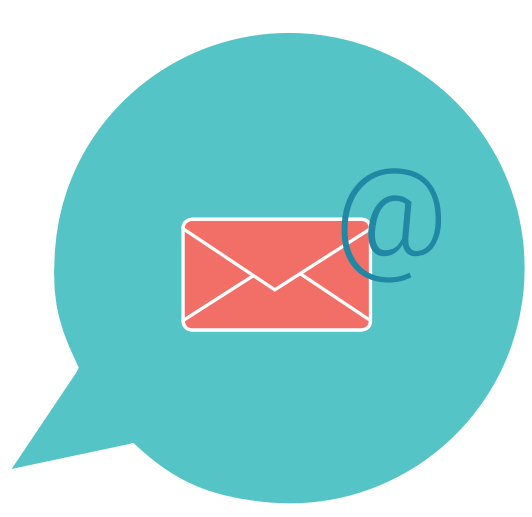

Alors qu'il y avait une pub très alléchante (conférence large avec des sessions parallèles sur différentes sous-disciplines de la chimie avec soi-disant de nombreux participants et des conférenciers de renom), je me suis retrouvée dans une petite salle minable (on ne voyait rien depuis le fond, pas de micros ni de pointeurs) d'un hôtel à Noisy le Grand (la conférence était annoncée à Paris), avec au max 20 personnes dans la salle. Programme totalement hétéroclite avec quelques rares conférences (de 20 minutes !) correctes et beaucoup de présentations sans aucun intérêt (et bien moins bien présentées que les présentations de L3 de nos étudiants).

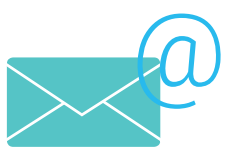

J'ai été effectivement à cette série de conférences, en 2016. Je vous déconseille toutefois de vous y rendre : prix d'inscription exorbitant, tous les frais sont à votre charge alors que vous êtes invité, le nombre total de personnes devait s'élever à 30 . Il n'y a pas vraiment de thématiques, c'est très très large.

La conférence avait lieu à San Francisco dans le centre de conférence Hilton (ce qui n'était pas désagréable et a certainement participé inconsciemment à ma volonté d'y aller), mais j'ai vite compris en arrivant que leur but n'était pas scientifique mais de faire de l'argent ! Le programme des conférences n'était pas inintéressant, mais il n'y avait évidemment pas de "cador " de la thématique ce qui fait que je n'ai pas appris grand-chose...

\section{Extraits anonymisés de courriers électroniques autour de conférences prédatrices.}

En jaune : réponse d'une direction de laboratoire : mon premier signalement avait reçu une réponse lénifiante, ce qui m'a amené à décrire très précisément des participations à l'organisation d'autres conférences douteuses.

En turquoise : deux avis sur une même conférence.

En violet : avis sur deux conférences du même organisateur. 


\section{Les dangers}

- J'ai pu observer à petite échelle que les missions pour ces rencontres douteuses se comptent en centaines par an en France : c'est une délinquance astucieuse qui détourne de l'argent public, pour un préjudice de plusieurs $M €$.

- «Acheter » le statut de " conférencier invité " biaise l'évaluation institutionnelle [7], à la promotion, comme pour un recrutement temporaire d'un jeune chercheur étranger. Si ne sont pas exigés des détails conséquents sur la nature des « invitations " (financement, taille et composition du public...), les indélicats sont avantagés.

- Outre la publication, assez bénigne, des lubies de scientifiques "illuminés ", les industries sur la sellette (tabac, pétrole, climatoscepticisme...) recherchent une caution scientifique [8]. Il est facile, dans ces conférences et revues prédatrices, de prétendre contrebalancer des résultats gênants établis par la recherche, et d'instiller le doute chez les décideurs.

\section{Des frontières qui s'estompent}

Les conférences scientifiques à vocation commerciale pullulent aussi. Cette "zone grise » dépasse celle où la conférence scientifique est l'« ornement » d'une foire professionnelle. Comme au spectacle, des «vedettes" bien choisies peuvent attirer un public payant. $\mathrm{Ce}$ sont sans doute des personnes à formation scientifique (collègues à l'éthique élastique, anciens étudiants...) qui repèrent les "cibles " de ces invitations, effectuées sans patronage ou subvention d'institutions. Les chercheurs en vue refusent difficilement une quelconque "présidence ", car celle-ci aidera à l'impérieuse nécessité de "communication " vers les décideurs et financeurs. Déchargés des soucis d'organisation, ces scientifiques trouveront parfois un soutien de leur institution (logo, prêt de salles...). C'est en s'attribuant des droits d'inscription indécents, au final payés par des crédits de recherche, que le commerçant organisateur se rémunère.

Cette zone " grise " devrait interroger notre pratique des conférences. Celles-ci se multiplient, car le chercheur dédaigne rarement une occasion de voyager : il y a l'espoir de trouver enfin «son " public et la reconnaissance afférente, par un facile recyclage de résultats, chèrement acquis et ne donnant lieu qu'à quelques publications originales. De façon circulaire, l'inflation de publications conduit à une lecture superficielle : on espère rattraper la bibliographie en allant en conférences. Pourtant, la plus-value propre aux réunions n'est patente que si les échanges et débats sont vraiment de qualité, ouvrant à réseautage ou collaborations futures. Les conférences de prestige, inévitablement rares, sont donc celles où les stars du domaine restent jusqu'au bout, assistent à plus que la demi-journée de leur session, et contribuent aux échanges. A contrario, la multiplication des sessions parallèles n'est pas un bon signe.

\section{Des réponses institutionnelles décevantes}

C'est en mentionnant les risques juridiques (" suspicion de fraude financière ") que j'ai pu faire retirer, nolens volens (cf. encart jaune de la figure 1) le soutien institutionnel à une conférence où j'apparaissais en coauteur. Sinon, prenant sur moi d'avertir des directions que leur laboratoire paraissait associé à des événements douteux (et peut-être par d'indélicats collègues), je n'ai eu que le silence en écho. Les témoignages individuels ont été plus faciles à obtenir (fig. 1).

Pire encore : des éditeurs de sociétés savantes, alertés de façon circonstanciée à propos de "Proceedings" très douteux, préferrent le déni. Pourtant, l'analyse de certains des ouvrages publiés (préface vague, nombre prétendu de participants ou contributions sans rapport avec les photos en ligne, non-publication des interventions "invitées »), et une comparaison avec les informations restées en ligne, sont édifiantes. Je tiens à disposition mes correspondances avec l'Institute of Physics britannique et l'American Institute of Physics, sans parier sur la rigueur des autres éditeurs spécialisés. Des " prédateurs » bien connus ou plus discrets se nichent toujours dans les annonces (gratuites) de "Natureevents", même si la revue Nature a promis d'exclure les conférences que j'avais signalées spécifiquement.

\section{Des propositions de remèdes}

De même que les DiSI (Directions des Services Informatiques) diffusent largement au sujet des virus et menaces, l'organisation d'un signalement issu des annonces suspectes permettrait de recenser, par l'Inist (Institut de l'information scientifique et technique du CNRS) par exemple, une liste noire des opérateurs de conférence non éligibles à mission. C'est une politique envisagée par la Chine [9] contre les journaux prédateurs.

La participation à ces évènements prédateurs dénote aussi, malheureusement, une certaine irresponsabilité individuelle. Si le financement pour une conférence était conditionné à une trace visible (la plateforme en ligne HAL ou équivalent), le risque de rester associé à une «invitation » indigne aiderait sans doute à la vigilance.

Enfin, plus globalement, la mise en ligne systématisée des rapports de relecteurs, proposée désormais par quelques revues, combattrait l'arnaque au "peer reviewing", commune aux "prédateurs ", journaux et conférences.

Daniel Bloch (daniel.bloch@univ-paris13.fr) Laboratoire de Physique des Lasers, UMR 7538 du CNRS, Université Paris 13 - Sorbonne Paris Cité 99 Avenue J.-B. Clément, 93430 Villetaneuse

Remerciements à Michèle Leduc, Charles Desfrançois, Ariel Levenson, qui m’ont encouragé à persévérer dans ces investigations à la frontière de la science.

\section{Références}

1• J. Beall, Nature 489 (2012) 179 ; Nature 534 (2016) 326.

$2 \cdot$ Cf. par exemple les articles de Wikipedia à propos de "Jeffrey Beall", "Predatory Publishing", "Predatory Conference", "WASET", "OMICS", "BIT Life Sciences", ou EMN_meetings/Research Gate.

3. K. Carey, "A Peek Inside the Strange World of Fake Academia", The New York Times (29 décembre 2016).

4. K Cobey et al., éditorial de Journal of Oncology Practice 13 (2017) 410.

5. D. Bloch, The Conversation, article 86957, 22 décembre 2017.

6. L.C. Martini, J. Phys: Conference Series, 495 (2014) 012001, 012002 et 012003.

7• J. Grove, Times Higher Education, 23 décembre 2017.

8• S. Chapman, The conversation, article 62497, 18 juillet 2016.

9• Nature (éditorial), 558 (2018) 162. 